\title{
BrainLock: A Generic Mu Rhythm based Locking System
}

\author{
Gove Nitinkumar Rajendra \\ Dept. of Computer Engg. \\ MIT COE, Pune, India
}

\author{
RajneeshKaur Bedi \\ Dept. of Computer Engg \\ MIT COE, Pune, India
}

\author{
Bhor Rohan Tatyaba \\ Dept. of Computer Engg. \\ MIT COE, Pune, India
}

\begin{abstract}
Every human activity is controlled and directed by brain. For each such activity there is equivalent action potential which gets generated. This action potential has a corresponding low power electrical signal. For different human actions different electrical signals are generated. This paper gives a definitive study of brain ' $\mu$ rhythms' which are generated during motor imagery activities. The current applications of these rhythms are also briefly elaborated. We also propose a generic mu rhythm based locking system which can be used for home security, bank locker security, vehicle locking and at many other places.
\end{abstract}

\section{General Terms}

EEG, Brain mu rhythms, Human-Machine Interface, Neuroprothetics

\section{Keywords}

Brain Waves, $\mu$ waves, BCI, HMI, User Interfaces

\section{INTRODUCTION}

Brain waves are the electrical signals which are generated for each action performed by a human. Brain waves are produced by the neurons and synapses which are part of central nervous system, during the course of their operations [1]. The variation in the surface potential distribution over the scalp reflects functional activities emerging from underlying brain [2]. These variations in surface potential distribution can be recorded by attaching an array of electrodes to the scalp and then measuring the voltage between pair of these electrodes. The signals obtained thus are filtered, amplified and analyzed to conclude the functions that obtained signal indicate.

According to the variations in the frequency ranges of brain waves, they are categorized in following five categories [3] [4]:

Delta rhythms: These waves have frequency within the range of 0.5 to $4 \mathrm{~Hz}$, with variable amplitude. These brain waves are primarily obtained during deep sleep.

Theta rhythms: These waves have frequencies within the range of 4-7 Hz with amplitude generally greater than 20 $\mu \mathrm{V}$. These waves are obtained during emotional stresses like frustration or disappointments.

Alpha rhythms: These waves lie within the frequency range of $8-13 \mathrm{~Hz}$, with $30-50 \mu \mathrm{V}$ amplitude. These waves generally indicate relaxed awareness and inattention.

Beta rhythms: These waves have frequencies in the range of 13-30 Hz. These waves are associated with active thinking, active attention, focusing something, concrete problem solving.

Gamma rhythms: These waves have frequency range of $35 \mathrm{~Hz}$ and above. These waves are associated with the mechanism of consciousness.

These different types of brain waves can be used in a variety of medical and scientific applications to automatically detect the mental state of the patient or the subject.

\section{BRAIN $\boldsymbol{\mu}$ RHYTHMS}

Brain $\mathrm{mu}(\mu)$ rhythms are the brain waves with frequency in the range of $8-13 \mathrm{~Hz}$ and $15-25 \mathrm{~Hz}$ [5]. These waves are also called as 'wicket rhythms'. Mu waves are generated during motor imagery activities i.e. when a person imagines himself of performing some activity. These waves diminish with movement or intent to move [4]. These waves are limited in a short range period of 0.5 to $2 \mathrm{~s}$ time [6]. They can be recorded over the sensorimotor cortex area of the brain when no activity is being performed by the subject.

Generally, the frequency range of mu rhythms overlap with the frequency range of occipital or classical alpha rhythms, so it becomes difficult perform differentiation between them. Figure 1 shows brain mu rhythms.
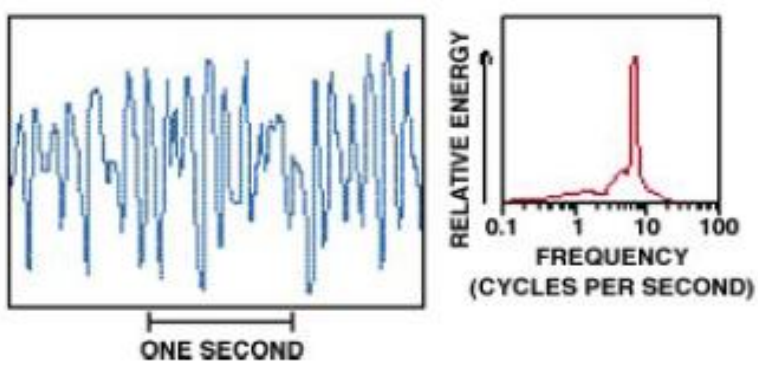

Figure 1. Mu waves

As already stated, frequency range of mu rhythms are similar to frequency range of alpha waves. Differentiation between these two kinds of waves is stated below: [7] 


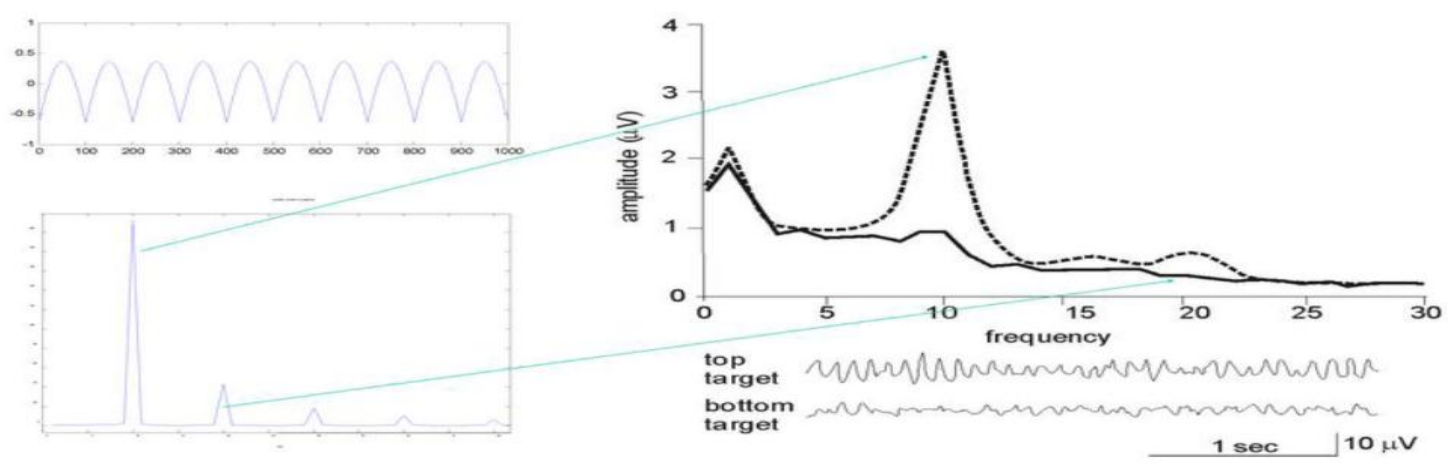

Figure 2 Temporal properties of brain mu rhythms

- $\quad \mathrm{Mu}$ rhythms do not exhibit bilateral coherence. While alpha rhythms exhibit bilateral coherence [8].

- Amplitude or power in the classical alpha rhythm is larger than that in mu rhythms.

- $\mathrm{Mu}$ rhythms exhibit more anterior focus compared to motor posterior one for the classical alpha waves.

- Generally, mu waves reflect sensorimotor processing in front parietal networks; on the other hand classical alpha reflects primarily visual processing in occipital networks.

- Mu rhythms are not affected by opening or closing of the eyes. While alpha waves are synchronized and attenuated when eyes are open.

Figure 2 shows the temporal properties of mu rhythms. This figure is taken from BCI2000 system's informative site which gives a definitive study of mu rhythms, BCI2000 system, BCI2000 experimental setups, and directions to be followed during experiments. It also gives information about Matlab libraries and implementations for implementing systems using BCI 2000. Mu rhythms generally have arc like shape (top left figure). In frequency spectrum this corresponds to a line spectrum with strong first harmonic (bottom left). This indicates that there will be a second peak in the beta band located at exactly twice the frequency of the first peak. The power of mu rhythms recorded from electrodes at scalp locations over sensorimotor cortex is reduced in normal age adults by self-initiated movement, imagined movement, and observed movement $[9,10,11$, and 12].

\section{APPLICATIONS OF $\mu$ RHYTHMS}

Over the past twenty five years, various studies have proposed and evaluated the possibility of brain signals being used as a new augmentative methodology/technology which does not require muscle control and applicability of this technology (e.g. [13]-[18]); a comprehensive study can be found in [19].

Brain $\mu$ (mu) rhythms have been studied and applied in various neuroprosthetic, brain signal controlled applications. $\mu$ rhythms have been used extensively in 1-D/2-D cursor control applications. The BCI developed at Wadsworth center enables user to control 1-D/2-D cursor movement on video screen using brain mu rhythms that are generated over sensorimotor cortex area of the brain. A comprehensive study and analysis of how mu $(\mu)$ rhythms can be used to control 1-D and 2-D cursor movement can be found in [2028]. $\mu(\mathrm{mu})$ rhythms are generated during motor imagery activities. Motor imagery is used many times during training activities by athletes and musicians for improving their performances. A repetitive activation of motor networks characteristics for a particular movement sequence may lead to strengthening of synaptic transmission in neurons in the same way as it has been shown for motor execution training.

Studies reveal that the better the imaging the better the training effect of mental practice [29]. Professional players use $\mu(\mathrm{mu})$ rhythm based imagery strategies frequently for improving their performance [30]. The applicability of $\mu$ (mu) rhythms automating piano is also studied and proved [31]. The usage of these rhythms for data encryption has also been proposed [32].

Apart from these widely studied applications, $\mu$ rhythms can be used in brain controlled home automation, robotic arm control, game playing, thought controlled machines development and other kinds of human machine interfaces(HMI)

\section{THE PROPOSED SYSTEM}

Based on the study of mu rhythms done, we propose a new brain rhythm based locking system. The three of the basic system operations are as explained below:

ENROLL: In this step, user uses a specific thought/imagined action to generate the key that will be used during system operation. The key generated for this specific user thought is then stored in key database. This key is referred during the other two system operations namely LOCK and UNLOCK. 


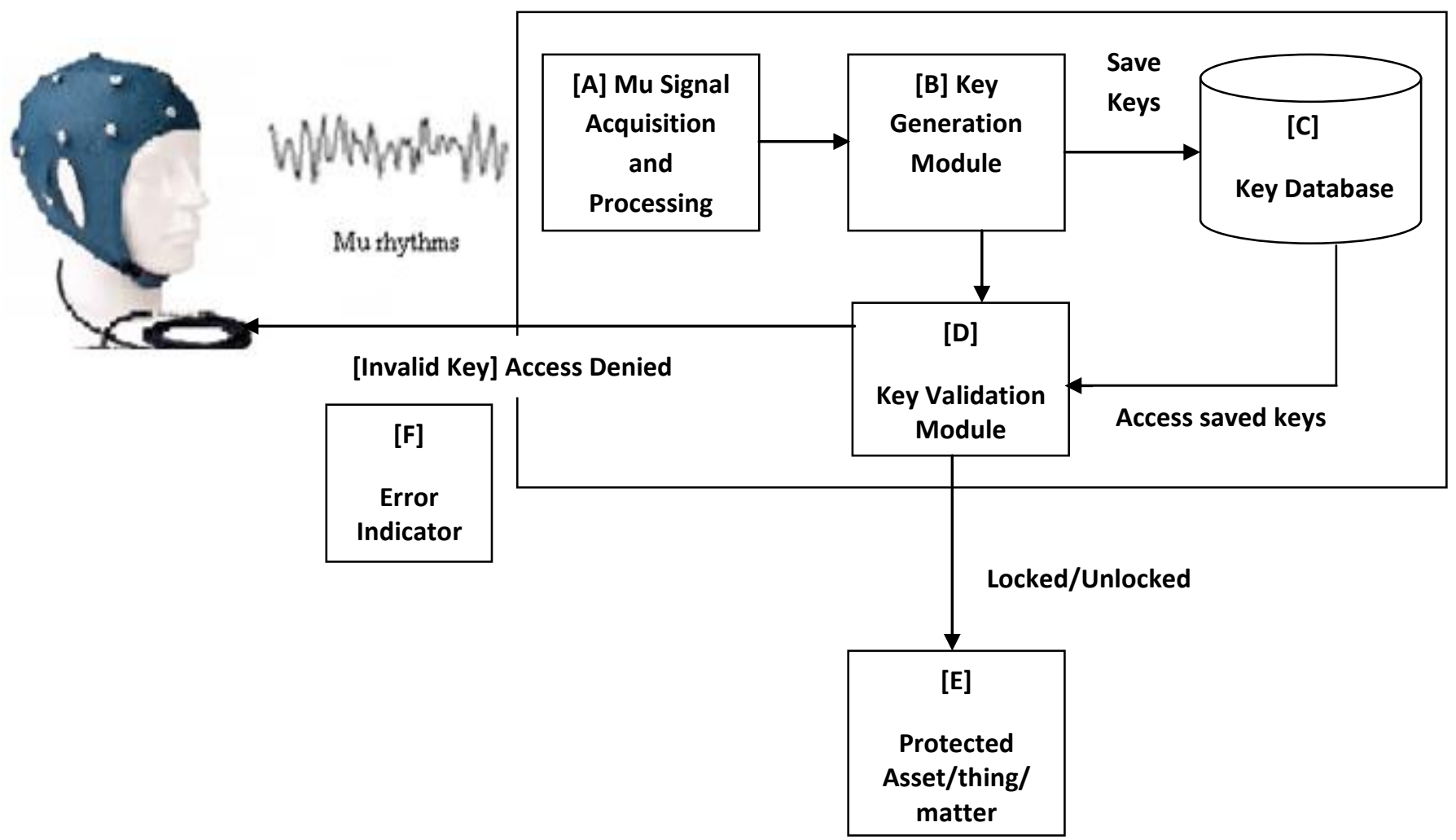

Figure 3 Block Diagram of BrainLock

LOCK: During this operation, the user thinks of the specific thought/imagined action which he used to generate the key during ENROLL operation. This user thought is then used to generate the actual key which then is used to secure the system by locking it

UNLOCK: This operation is used to re-access/unlock the asset or things that are protected by the 'BrainLock'. Here, a user has to rethink his thought which is then used to generate the key. The newly generated key is then checked with the key that is already stored in the key database. If both the keys match, the protected system gets unlocked.

During successful execution of UNLOCK operation the sequence of blocks that get executed is A-B-C-D-E. On unsuccessful execution of UNLOCK operation following sequence of blocks get executed: A-B-C-D-F. The block diagram of the proposed system is as shown in figure 3. As depicted in it, the main components of the proposed system are:

1. $\mathrm{Mu}$ rhythm acquisition and processing module[A]

2. Key Generation module[B]

3. Key Database[C]

4. Key Validation Module[D]

5. Protected asset/things/matter[E]

6. $\quad$ Error Indicator[F]

Each of these modules is briefly described below:

Mu rhythm acquisition and processing module: This module is responsible for capturing the human thinking in the form of an EEG signal. The signal captured thus is then preprocessed to remove noise, artifacts and other outliers from it. This processing gives us the actual mu rhythms generated for the human thought. Once this is done, spike sorting, feature extraction is done. The extracted features are then used by the key generation module for generating a unique key.

Key Generation module: This module generates a unique key based on the features received from the previous module. Once the key is generated, this module stores it into the key database for latter reference during system LOCK and UNLOCKS operations.

Key Database: This is a key repository. Basically, this is database of keys that are generated by the system for particular users thought[s]. The amount of information stored in this database may vary over different system implementations and applications. For this purpose the key database design needs to be customizable.

Key Validation Module: This module is used to validate the user access to the protected system. When a user wants to access the system, he has to rethink the thought that he has kept as key. A key is generated for this thought and is checked against the one stored in the database during ENROLL operation. If the key is valid, user is granted with the access to the system. Else 'BrainLock' denies access to the user. The denial of access to the protected system is indicated by glowing an error indicator LED.

Protected asset/things/matter: This is the actual user asset, thing, or matter that is being protected by 'BrainLock'. It can be user's home, bank locker, car etc.

Error Indicator: This is a simple LED which is used to indicate the occurrence of some error in the system. Most of the times it is used to indicate use of invalid key to access the asset/things protected using 'BrainLock'. The other conditions which can be indicated using this simple component can be power failure, system error etc. 


\section{CONCLUSION}

This paper provides a comprehensive study of mu waves and also specifies some of the real life applications of these waves. Study reveals that these waves can be used to capture the human imagination. An approach for asset/thing security is also proposed in this paper based on the mu rhythm study done. The proposed system provides a user with the freedom of using any of his thought as key to lock and unlock the system. As it is very difficult to guess human thoughts this system will surely provide a very high amount of security to user's assets and things. In future, we will look forward for successful implementation of the efficient 'BrainLock' system with improved efficiency and easier user access.

\section{ACKNOWLEDGEMENTS}

We thank Department of computer engineering, MIT COE, Pune, India for their kind support.

\section{REFERENCES}

[1] Georg E. Fabiani, Dennis J. McFarland, Jonathan R. Wolpaw, GeOE ,Pfurtscheller ,Conversion of EEG activity into cursor movement by a brain-computer interface(BCI), IEEE transactions on neural systems and rehabilitation engineering, Volume 12 No. 3 ,September 2004

[2] E. R. Kandel, J. H. Schwartz, and T. M. Jessell, Principles of Neural Science, 3rd Ed. New York: Elsevier/NorthHolland, 1991.

[3] Jorge Baztarrica Ochoa, Gary Garcia Molina, Touradj Ebrahimi .EEG Signal Classification for Brain Computer Interface Applications, ECOLE POLYTECHNIQUE FEDERALE DE LAUSANNE, March $28^{\text {th }}, 2002$

[4]A. Tzelepi, T. Bezerianos, I. Bodis-Wollner, "Functional properties of sub-bands of oscillatory brain waves to pattern visual stimulation in man", clinical neurophysiology volume 111, Issue 2, 1 February 2000, Pages 259-269

[5] R. Hari, R. Salmelin, Human cortical oscillations: a neuromagnetic view through the skull, Trends Neuroscience. 20 (1997) 44-49

[6] E. Niedermeyer, A. Goldszmidt, D. Ryan, "Mu Rhythm Status" and clinical correlates, Clinical EEG Neuroscience. 35 (2004) 84-87

[7] Jaime A. Pieda, The functional significance of mu rhythms: Translating "seeing" and "hearing" into “doing”, Brain Research Reviews 50 (2005) 57- 68

[8]W. Storm van Leeuwen, A. Arntz, P. Spoelstra, G. H. Wieneke, The use of computer analysis for diagnosis in routine electroencephalography, Revised Electroencephalography. Neurophysiology. Clinical. 6 (1976) 318-327

[9] C. Babiloni, F. Carducci, F. Cincotti, P.M. Rossini, C. Neuper, G. Pfurtscheller, F. Babiloni, Human movementrelated potentials vs. De synchronization of EEG alpha rhythm: a high-resolution EEG study, Neuro image 10 (1999) 658-665.

[10] S. Cochin, C. Barthelemy, B. Lejeune, S. Roux, J. Martineau, Perception of motion and qEEG activity in human adults, Electroencephalography. Clinical. Neurophysiology. 107 (1998) 287-295.

[11] H.J. Gastaut, J. Bert, EEG changes during cinematographic presentation, Electroencephalography. Clinical. Neurophysiology. 6 (1954) 433- 444.

[12] J.A. Pineda, B.Z. Allison, A. Vankov, The effects of selfmovement, observation, and imagination on mu rhythms and readiness potentials (RP's): toward a braincomputer interface (BCI), IEEE Trans. Rehabilitation. Eng. 8 (2000) 219- 222.

[13] L. A. Farwell and E. Donchin, "Talking off the top of your head: Toward a mental prosthesis utilizing eventrelated brain potentials," Electroencephalography. Clinical. Neurophysiology, vol. 70, no. 6, pp. 510-523, Dec. 1988.

[14] E. E. Sutter, "The brain response interface: communication through visually guided electrical brain responses," J.Microcomputer. Applications. vol. 15, pp. $31-45,1992$

[15] G. Pfurtscheller, D. Flotzinger, and J. Kalcher, "Braincomputer interface-A new communication device for handicapped persons," J. Microcomputer. Applications. vol. 16, pp. 293-299, 1993.

[16] N. Birbaumer, N. Ghanayim, T. Hinterberger, I. Iversen, B. Kotchoubey, A. Kubler, J. Perelmouter, E. Taub, and H. Flor, "A spelling device for the paralyzed," Nature, vol. 398, no. 6725, pp. 297-298, Mar. 1999.

[17] A. Kubler, B. Kotchoubey, T. Hinterberger, N. Ghanayim, J. Perelmouter, M. Schauer, C. Fritsch, E. Taub, and N. Birbaumer, "The thought translation device: A neurophysiological approach to communication in total motor paralysis," Exp. Brain Res., vol. 124, no. 2, pp. 223-232, Jan. 1999.

[18] P. R. Kennedy, R. A. Bakay, M. M. Moore, and J. Goldwaithe, "Direct control of a computer from the human central nervous system," IEEE Trans. Rehab. Eng., vol. 8, pp. 198-202, June 2000.

[19] J. R.Wolpaw, N. Birbaumer, D. J.McFarland, G. Pfurtscheller, and T. M.Vaughan, "Brain-computer interfaces for communication and control," Electroencephalography. Clinical. Neurophysiology. vol. 113, no. 6, pp. 767-791, June 2002.

[20] D. J. McFarland, G. W. Neat, R. F. Read, and J. R. Wolpaw, "An EEG based method for graded cursor control,Psychobiological” vol. 21, pp. 77-81,1993.

[21] J. R. Wolpaw and D. J. McFarland, "Multichannel EEGbased brain computer communication," Electroencephalography. Clinical. Neurophysiology. vol.90, pp. 444-449, 1994.

[22] J. R. Wolpaw and D. J. McFarland, "Control of a twodimensional movement signal by a noninvasive braincomputer interface in humans," Proc. Nat. Acad. Sci. USA (Pub 2004), vol. 101(51), pp.17849-54, Dec. 21, 2004

[23]Wolpaw, J.R., McFarland, D.J., Neat, G.W. and Forneris, C.A. (1991). An EEG-based brain computer interface for cursor control. Electroencephalography Clinical Neurophysiology, 78, 252-259. 
[24] Wolpaw, J.R. and McFarland, D.J. (2003). Twodimensional movement control by scalp-recorded sensorimotor rhythms in humans. Program No. 607.2. 2003 Abstract Viewer/Itinerary Planner. Society for Neuroscience, Online, Washington, DC.

[25] McFarland DJ, Miner LA, Vaughan TM, Wolpaw JR. "Mu and beta rhythm topographies during motor imagery and actual movement". Brain Topographies 2000a; $3: 177-186$

[26] Wolpaw JR, Flotzinger D, Pfurtscheller G, McFarland DJ. Timing of EEG based cursor control. Clinical Neurophysiology 1997; 16: 529-538.

[27] Vaughan TM, Sarnacki WA, McFarland DJ, Wolpaw JR. EEG-based communication with topographically differentiated $\mathrm{mu}$ and beta rhythms. Society of Neuroscience Abstract 1999; 25:1412.
[28] Bhor Rohan T., Kad Reshma H., Katariya Payal J., Rajneesh Kaur Bedi, Gove NitinKumar R.,"Motor imagery for mouse automation and control", IJCSIS October 2011 issue.

[29]Martin Lotze, Ulrike Halsband, Motor Imagery, Journal of physiology, Paris, 99(2006) 386-395

[30] Cumming J., Hall C., 2002 deliberate imagery practice: the development of imagery skills in competitive athletes. J. Sports Sci. 20, 137-145

[31] Bangert M., Haeusler U., Altenmuller E. 2001, on proactive: how the brain connects piano keys and piano sounds. Ann. N.Y. Acad. Sci. 930, 425-428.

[32]Gove Nitinkumar Rajendra, Bedi RajneeshKaur, "A new approach to data encryption using genetic algorithms and brain mu waves", IJSER May 2011 issue. 